\title{
A Comprehensive Study of the Trends and Analysis of Distributed Ledger Technology and Blockchain Technology in the Healthcare Industry
}

\author{
Anusha Thakur* \\ University of Petroleum and Energy Studies, Dehradun, India
}

In today's scenario, it is essential for the healthcare sector to focus on balancing patient care records with information relevant to completeness, accessibility, and privacy concerns. Advancements in information technology and health infrastructure exponentially bolster transformative changes in the healthcare industry. Incorporation of blockchain along with distributed ledger technology (DLT) owes the potentiality to cater to the interoperability restraints in health IT systems and enables medical researchers,

OPEN ACCESS

Edited by:

Tsung-Ting Kuo,

University of California, San Diego,

United States

Reviewed by:

Pavlos Papadopoulos, Edinburgh Napier University,

United Kingdom

Alexander Norta,

Tallinn University of Technology,

Estonia

*Correspondence:

Anusha Thakur

anushathakur89@yahoo.com

Specialty section:

This article was submitted to Blockchain Technologies, a section of the journal

Frontiers in Blockchain

Received: 28 December 2021

Accepted: 03 February 2022

Published: 03 March 2022

Citation:

Thakur A (2022) A Comprehensive Study of the Trends and Analysis of Distributed Ledger Technology and

Blockchain Technology in the

Healthcare Industry.

Front. Blockchain 5:844834.

doi: 10.3389/fbloc.2022.844834 healthcare entities, and healthcare providers to share electronic health data in a secured and well-mannered system. In addition to these, such technologies also propose and offer latest models for health statistics exchange by making the records more secure and efficient. However, successful implementation of blockchain technology and DLT necessitates efficient infrastructure, connectivity, and other factors. Hence, there poses to be several challenges restraining the mainstream usage of blockchain technology in the healthcare sector. The article illustrates different generations of blockchain, issues in healthcare data, and network structures as well as the solutions offered by the sector to cater to such problems. In addition to these, the article also emphasizes on the different application areas of blockchain and DLT in healthcare infrastructure. This article further discusses latest trends and factors driving the need for the incorporation of blockchain and distributed ledger technology in the healthcare sector and the future scenario for the same.

Keywords: blockchain technology, distributed ledger technology, healthcare, records, information

\section{INTRODUCTION}

Blockchain was initially applied as a fundamental technology for the cryptocurrency "Bitcoin", which has budding usage beyond the realms of cryptocurrencies and digital currencies. Blockchain is based on the concept of "append-only" open ledger, according to which every contract occurring within a setup, is added to the ledger, only when verified by the connections in the system (CapGemini, 2017).

The technology is evolving as one of the promising technologies which acquire the attention of different industries and research backgrounds. Blockchain is a shared distributed ledger technology (DLT) which minimizes the need for an intermediary to manage transaction and minimize the cost and chances of malpractices in the system (Yadav, 2019). The features of this technology, delivered via composed and cautious blend of in-built enticements and effective cryptography, exclude the demand for a centrally ruling organization and instead circulate the power among all contributors in the blockchain ecosystem (FourWeekMBA, 2021). 
One of the fields wherein blockchain is gaining exponential traction is the Figure $\mathbf{1}$ healthcare industry. This concept owes the potentiality to completely transform the healthcare segment by focusing on the needs of the patients and accordingly increasing the interoperability, privacy, and security of the healthcare-related information. Adoption of this technology in the healthcare sector enhances the healthcare practices, for instance, the quality of patient care and healthcare service delivery (Prokofieva and Miah, 2019). Growing pertinence of the technology is expected to open several avenues in various aspects of the industry, such as the advancement in medical research and wearables segment as well.

Adoption of blockchain by the healthcare businesses helps to cater to several issues such as permitting the accessibility of health data by the patients, EHR interoperability, and creating trust in between the healthcare providers, in terms of privacy and auditability. Blockchain provides seamless sharing of information, thereby minimizing inconsistencies and errors, due to the storage of centralized data.

\section{Research Questions}

- Need for blockchain technology in the healthcare industry?

- How is blockchain poised to bolster the business models?

- What are the growth opportunities and trend analysis for the blockchain technology in healthcare sector?

- Top-five use cases within the healthcare segment which owes the capability to completely transform the healthcare sector.

\section{Purpose of the Article}

This article illustrates the different factors bolstering and hindering the market of blockchain technologies in the healthcare sector. The article emphasizes on key opportunities for the application of these technologies in the healthcare sector. In addition to these, the study also includes a brief description of the top five use-cases illustrating the solutions and competitive strategies adopted by the existing players and new entrants catering to different issues in the healthcare space.

\section{Key Findings in the Research}

The article focuses on how efficiently cutting-edge technologies such as blockchain transforms the dynamics of the healthcare sector. It presents an in-depth description of the factors bolstering the demand, along with different challenges and opportunities, impacting the adoption of blockchain in the healthcare sector.

\section{LITERATURE REVIEW}

The blockchain technology has significantly evolved over the years and is expected to revolutionize the wide-range of information-driven segments. Blockchain refers to a chain of transactions as blocks associated together by cryptographic signs, each of which is known as a hash, stockpiled in joint ledgers, and supported by a system of linked processes called nodes (Healthcare Information and Management Systems Society, 2021).

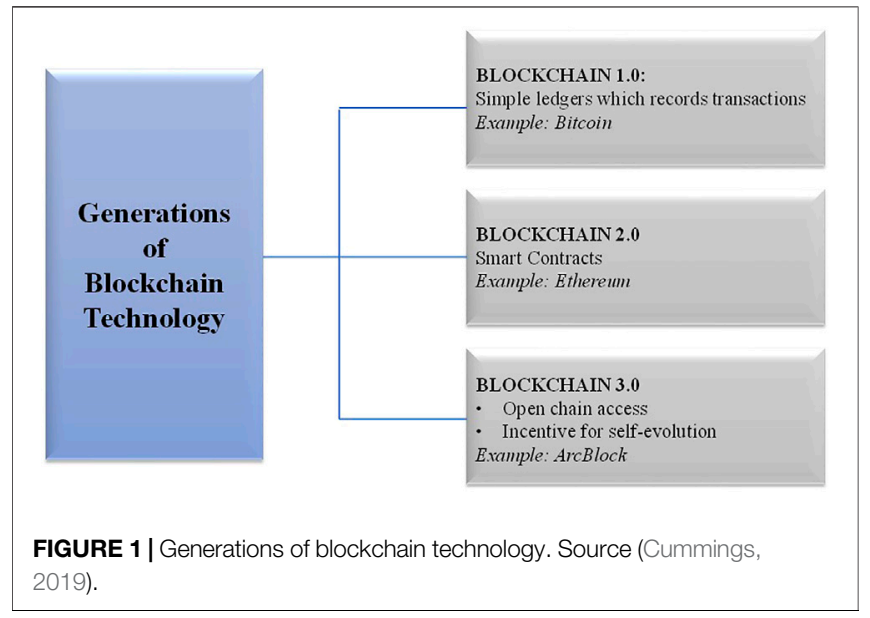

The technology is a public record made up of a system of blocks, which grasps the entire transactional histories occurring within the system (Ben Fekih and Lahami, 2020).

\section{Generations of Blockchain Technology}

- The first generation (Blockchain 1.0) is the execution of blockchain on the basis of cryptocurrency usage to radically enhance the monetary systems. These are based on the proof-of-work algorithm.

- The second generation (Blockchain 2.0) is also based on the proof-of-work algorithm; however, these have extensive functionality in comparison to the Blockchain 1.0. For instance, "Ethereum blockchain" owes the potentiality to maintain the decentralized applications and processes smart contracts (Cummings, 2019).

- The third generation (Blockchain 3.0) deals with nonfinancial application segments, which include healthcare, energy sector, and government sector.

The emergence of this technology from blockchain 1.0 to blockchain 4.0 generation has been illustrated in the following figure:

\section{CURRENT HEALTHCARE SYSTEMS}

Healthcare is a huge market worldwide. In the United States alone, the total healthcare expenditures were estimated to be approximately $\$ 3.55$ trillion in 2017 and are predicted to reach more than $\$ 5.5$ trillion in 2025 . However, this important sphere of our life is considered extremely inefficient. The Institute of Medicine estimates that between 20 and 30 percent of total healthcare spending being either wasteful or a consequence of poor outcomes.

In today's scenario, revolution in the healthcare industry mainly emphasizes on the adoption of patient-centric models, wherein, healthcare businesses are engaged in offering the bestin-class experience to their patients at an optimal cost. In addition to this, the government in several regions are making it necessary nowadays that health information exchange (HIE), Medicaid 
expansion, and health insurance marketplaces emphasize toward attaining "patient care" as the key focus area in their healthcare delivery systems (CapGemini, 2017).

\section{FACTORS BOLSTERING THE NEED FOR BLOCKCHAIN TECHNOLOGY IN THE HEALTHCARE SYSTEM \\ Drug Counterfeiting}

One of the key issues in the healthcare industry is counterfeiting of drugs. Counterfeited medicines often cause threat to the safety and health of the patients, thereby posing to be wastage of income for the same (Blackstone et al., 2014).

The cases of counterfeiting are significantly increasing across different regions in the world and have infringed the drug supply chain in countries such as the US. Nearly 10-30 percent of the medicines being sold in emerging countries are counterfeited. In addition to these, approximately 80 percent of fake drugs consumed in the US come from abroad (Health Research Funding, 2021). The issues of counterfeiting exist in the countries of North America and Europe, despite inclusion of stringent inspection and law enforcement programs by different drug regulatory agencies. In addition to these, countries with the maximum number of counterfeiting incidents are not necessarily to have lower law enforcement and weaker regulations. In developing countries, counterfeiting medicines are available almost for all diseases, whether it is for antimalaria or cough-related issues. Furthermore, in lesser income countries, wherein, regulatory and legal oversights are not so properly defined, the conditions are even worse, with counterfeited drugs being available for life-threatening situations such as tuberculosis, AIDS/HIV, and malaria (Pharma IQ News, 2011).

\section{Data Storage and Security}

Privacy and security are crucial factors for effectual access control models in the healthcare structures (Ali et al., 2021). Handling large-scale information and preserving the privacy of patients pose to be a challenge for the researchers (Onik et al., 2019).

In addition to these, misuse of the healthcare information is also another issue with the system. For instance, not all the clinical reports reported in the US means loss of data. At times, the information gets sold to the third-party firms. Poor handling of information can be attributed to the usage of outdated systems such as data silos which are not connected with the healthcare applications and systems. In addition to these, it becomes difficult to find related information for few healthcare platforms, since they have opted for everything to be local. This is expected to be time-consuming for the doctors at the time of data search and costly for the patients. Implementation of blockchain technology ensures ease of management and security for sensor-based information. Hence, it is becoming essential for the healthcare systems to implement blockchain technology in their businesses, to cater to the concerns of data security and storage.

\section{No Proper Management and Data Segmentation}

Maintenance of healthcare information poses to be another aspect wherein performance of the healthcare sector proves to be frail. Critical information about the patients disorganized all over the department and systems, with no way to get the required information at right time, might at times cause delay in providing information to the doctors as and when they need it. Even, it poses to be challenging for the patients to manage their reports at times.

Hence, due to lack of information available, healthcare systems at times fail to offer vital treatment to the patients, thereby impacting their health and safety (Blockchains, 2019).

\section{FACTORS RESTRAINING THE ADOPTION OF BLOCKCHAIN IN HEALTHCARE}

Blockchain is still in its developing stage, particularly for the healthcare sector, with several challenges being faced in deployment of the same. For the blockchain technology to work efficiently, organizational, governance, and technical segments are expected to play a vital role. It is therefore vital for the blockchain approaches to be responsive, with respect to the changing demands from different viewpoints of providers, regulators, and patients (Attaran, 2020).

- Technical: Blockchain is still in its nascent stage wherein issues, such as non-standardization, non-scalability, and integration with current legacy systems, need to be addressed upon. Few other technical issues in the adoption of this technology includes the need for a system of interrelated nodes to provide necessary processing power, to generate blocks upon the submission of a transaction, along with the unseen cost of operating the same.

- Organizational: Incentives used to generate a widespread network of connected data blocks as a record-keeping agenda to replace the traditional system has not been there yet. Key organizational challenges include unknown cost of blockchain, along with certain interoperability issues.

- Regulatory policies: Uncertainty in regulations around immature policy or legal landscape for distributed ledger technology is expected to limit the adoption of blockchain technology on a larger scale.

\section{ANALYSIS OF GROWTH OPPORTUNITIES OF BLOCKCHAIN IN HEALTHCARE}

Blockchain technology is gaining significant traction in the market owing to its data-handling capabilities in terms of security and decentralization factors. The technology has witnessed profitable implementation in the financial sector and is now emphasizing on exploring the potentialities in other industry domains as well. 
With the changing scenario, it becomes essential for the healthcare sector to equip itself and offer best possible services and solutions. In today's scenario, pharma companies, healthcare providers, and insurance payers are increasingly the adopters of blockchain technology. Most of the drug wholesalers and manufacturers are engaged in discovering the potentialities of the technology in terms of government policies and regulations, to cater to the shifting trends. The following figure illustrates a brief snapshot of different applications of blockchain and the companies involved in the same.

\section{Claim Management and Healthcare Payment}

Problem: Claims and healthcare billing are full of fake activities, wherein the billing sector suffers the maximum. Hospitals pose to be the frontliners for the same, as they are engaged in billing the services which either have not been taken or are highly priced, in comparison to different industry standards. These activities are basically carried out for maximizing the overall profit, keeping the awareness of patients at bay.

Mediators within the systems designated to validate the claims are hesitant in their job, thereby leaving the end users clueless. The claims are expected to take a lot of time for processing and letting the individuals visit the office any time before it gets dealt with. In addition to this, intermediaries involved in the process guarantee and decide the say information and ensure the minimization of time lag for processing the claim. The conventional process of claim management comprises a lot of to and fro interaction between the parties for processing the claim. Increasing complexity of claim management and cost of claims are few of the significant challenges being faced by most of the health insurance players in today's scenario. Digitizing every step in the claim management process ranges from payment to data input and owes the potentiality to restructure and bolster the accuracy and efficiency of the same. Implementation of blockchain helps cater to such challenges at the time of processing the payment and claim adjudication activities.

Opportunities: Deployment of blockchain technology in the healthcare sector for application areas such as billing management, revenue cycle management (RCM), and claims adjudication play a pivotal role in enhancing the overall market growth. In the long-term scenario, decentralized healthcare payment and insurance models are projected to gain significant traction by exploiting DLT's protocol by accessing the patient's health-related information and accordingly sponsoring the patient-centric insurance programs. Implementation of blockchain technology is expected to cater to several inefficiencies with the help of transparent and trusted systems for the investors in businesses. These technological systems help streamline workflow communications and unlock the latest economic advantages by automating transactional services and restraining the intermediaries across healthcare services. By removing the intermediates, the technology enhances both billing and claim aspects of the healthcare industry. In case of the billing processes, healthcare providers get no chance to overcharge or add the services which have not been availed by the patients. In addition to this, the claim management process is expected to be reduced to a few minutes rather than months with the inclusion of blockchain technology.

Usage of a digital approach thereby enables the private payers to cater to several challenges, attain long-term vision of digitalization, and hence position themselves in a better way. For the payers, one of the key challenges in healthcare claim management includes minimization of operating and medical costs with improvement in consumer experience. Implementation of a digital approach for the claim processes helps the payers attain the path of digitization. Hence, innovation in three primary dimensions of claim management processes by the private payers is gaining traction nowadays. These are illustrated in the following figure (Source: (Singhal et al., 2019)):

Claim Procedures:

- Increasing pressure to minimize the traditional paper-based processes.

- Growing pressure to use technological advancements in processing the data to reduce the operating costs.

Interaction with the Customers:

- Growing demand for protection and transparency of data and their availability.

- Increasing need for enhancement of consumer experience.

- Adaptable product portfolios and strategies and product portfolios for latest technology.

Medical Expenses:

- Rising imperative for enhanced risk allocation.

- Lesser manually checked claims and medical costs.

Company 1:

Change Healthcare:

Change Healthcare, a US-based company, is one of the leading providers of clinical information exchange solutions, claim management, and RCM in the market. The expertise network of Change Healthcare provides an increasingly collaborative, well-coordinated, and efficient healthcare system. The key application segments of the company include clinical and imaging (helps optimize the financial performance), payments and revenue cycle (revolutionizing care and operational effectiveness), and member and patient engagement (improves the healthcare experience). The company operates with nearly 12 locations in approximately five different countries in the world (Change Healthcare, 2020).

Strategies adopted by Change Healthcare:

- In November 2018, the company announced its partnership with TIBCO Software for building a smart contract system via the Change Healthcare blockchain technology, hence enabling the partners to easily develop and install smartcontract processes which mechanize the events across healthcare transactional processing development. 
- In 2018, Change Healthcare announced the availability of first enterprise-scale blockchain solution for healthcare sectors, which is presently capable of processing nearly 50 million claim events every day. Incorporation of this solution enables the company to effectively manage daily national throughput requirements and transactional loads in comparison to the conventional insurance eligibility checks.

\section{Company 2:}

Solve.Care:

Solve.Care, a global healthcare platform, is headquartered in Singapore. The company is engaged in leveraging blockchain technology for the coordination, payment, and benefit administration of all investors in the healthcare segment. Solve.Care emphasizes on making the healthcare systems affordable, effective, and accessible. The company focuses on processing the payments and guarantees the elimination of additional billing by entities. Solve.Care is engaged in decentralizing the healthcare segment by tokenizing the transactions, data, consent, identity, and payments. Different components of the platform include Care.Wallet, Care.Vault, Care.Cards, Care.Ledger, Care.Protocol, and Solve. The company operates with its distribution facilities in the regions of South Korea, US, the United Kingdom, India, and Estonia.

Strategies adopted by Solve.Care:

- In October 2019, the company announced the incorporation of its headquarters in Singapore and its expansion in the Asia-Pacific region with its newly opened office in Seoul, South Korea.

\section{Healthcare Data Exchange}

Problem:

Health Data Ownership Issue: Growing consumer dataownership and awareness discussions are expected to increase with time. For instance, Government of Finland and Netherlands constituted that the individuals need to have access to their health records.

Health Data Interoperability Challenge: The present unified healthcare IT systems generate silos and intensify the error rates for integration and identification of the data files of patients.

Health Data Regulatory Changes: The current digital transformation of the healthcare industry with uneven healthcare connected and IT devices is expected to enhance susceptible end points for the healthcare data breaches.

Opportunities: Deployment of large-scale public health records (PHRs) applications poses to be more feasible in the Asian and European regions, wherein, the data vendor ecosystem is less complex than the market in the US. Incorporation of blockchain enables encryption of all the data in the network. Only few participants or nodes are allowed to access the data, hence maintaining the privacy and confidentiality of the information (CitiusTech, 2018).

The technology thereby poses to be a reagent for the digital roadmaps and their conjunction with other technologies, such as analytics, artificial intelligence (AI), and few more, to generate a decentralized learning system.

Company:

MediBloc Limited.

MediBloc is engaged in creating a patient-centric health data ecosystem, which protects the privacy of individuals and maximizes the reliability of health data, with the development of "Panacea" (a decentralized blockchain network to maintain the interoperability and sovereignty of health data). In a tamperproof and decentralized blockchain-data ecosystem, sovereignty of the healthcare data can be maintained.

Strategies adopted by MediBloc Limited:

- In December 2018, MediBloc announced the full version of its healthcare data platform for several applications such as information-sharing system, blockchain ID, cryptocurrency transaction, and PHR. The main platform has been tried across several PoCs conducted in collaboration with top medical businesses and institutions in South Korea.

Source: Company Website (MediBloc Limited, 2021)

\section{Pharmaceutical Counterfeit Control}

Problem: Production of counterfeit drugs in the pharmaceutical segment poses to be one of the major issues being faced, particularly in the developing regions, owing to the discrepancy in supply chain processes. Nearly 10 percent of the drug or pharma supply chain is counterfeited, which outlays approximately USD 200 billion annually (SA). Increasing e-commerce platforms, along with the globalization of consumer markets, is expected to result in increased penetration of forged medicines, including delivery and purchase of the same via online channels.

Opportunities: Incorporation of blockchain technology in the healthcare industry emphasizes on the proper understanding of the working process of ledger technology. Blockchain helps add security and traceability to the supply chain processes, thereby offering visibility to the drug regulatory authority and the manufacturers of the system (Haq and Muselemu, 2018). The purpose of the blockchain-based supply chain management process for the healthcare sector emphasizes on the following:

- Enhanced database for future scenario: Usage of blockchain in keeping the record of the patients poses to be safe in comparison to the conventional databases, wherein privacy and personal data of the patients are at risk.

- Extended security: Blockchain, one of the secured ledger systems, wherein the information stored on it, cannot be modified or deleted.

- Traceability: Adding of traceability enables the drug manufacturers to keep a check on the step-by-step supply chain processes, thereby increasing the authenticity of the product.

- Protect privacy and add visibility: Incorporation of blockchain in the pharmaceutical supply chain system allows the products to be verifiable without disclosing 
any information related to the techniques of the manufacturer.

Hence, implementation of blockchain technology provides excellent solutions to handle the counterfeiting issues. This technology offers immutability and transparency solutions in the supply chain processes. It enables the providers to:

- minimize theft issues and

- minimize counterfeiting issues.

Manage inventory (LeewayHertz, 2021)

Company 1:

AlpVision SA.

AlpVision SA is one of the leading players in the anticounterfeiting digital technologies in terms of counterfeit protection and product authentication. In 2001, AlpVision announced the invention of a breakthrough technology, broadly used by Forbes. The company is engaged in offering AI-based counterfeiting solutions in the areas of automotive, precious metals, chemistry, pharmaceuticals, tobacco, image analysis, quality control, and others. The solutions in different segments are commercialized across different regions in the world under the license agreements as completely tailored turnkey computerized arrangements (AlpVision SA, 2021).

Strategies adopted by AlpVision SA:

- In September 2016, AlpVision announced the release of smart embossing and new anti-counterfeiting technology.

- In 2015, AlpVision announced the extension of its business activities by offering artificial intelligence-based products to different application segments.

\section{Company 2:}

FarmaTrust.

FarmaTrust is engaged in innovating and digitizing the healthcare and pharmaceutical businesses. The company emphasizes on eliminating the counterfeiting of products, protecting the patients, and providing latest data analytical tools. The company emphasizes on offering end-to-end visibility and transparency across the supply chain processes in the pharmaceutical segments. FarmaTrust uses artificial intelligence and distributed ledger technology to create efficiencies and save the lives of patients in the healthcare sector. The key business segments offered by the company include pharmaceutical tracking and data, cell and gene therapies, and medical device services as well as vaccination solutions.

\section{Clinical and Research Trials}

Problem: Personal data privacy concerns, data sharing, reproducibility, and patient enrollment are few challenges in the pharma and medical clinical trial segment.

Limited secondary research due to underreporting of clinical trial data: According to the study, nearly 50 percent of the clinical trials go unreported, wherein the investigators often fail to share the results. This is expected to create knowledge gaps for the healthcare policy makers and investors as well as several vital safety concerns for the patients.

Engagement and recruitment of the clinical trials patient: Nearly 80 percent of the clinical trials do not meet the deadlines, thereby leading to an average loss of nearly USD 1.3 million per day for any individual. Due to the absence of patient-centric trial designs, approximately 35 percent of the patients opt out of clinical trials.

eSource data provenance and integrity for regulatory purpose: The sponsors for clinical trials spend nearly 25 percent of the entire budget for source data verification (SDV), to guarantee provenance and integrity for the gathered information for regulatory requirements. Growing preferences for the eSource data are expected to increase the process complexities and costs relevant to ethical management and data collection, reproducibility, and IT system integration processes.

Consent management to access individual real-world data (RWD) for research: The trial process is subjected to temporary protocol reforms, which is expected to make the patient consent collection a challenging and dynamic process. Growing trends of population research concepts are expected to create several concerns with the newest consent management method (Frost and Sullivan, 2019).

Opportunities: Inclusion of the DLT system in healthcare businesses ensures patient-centric solutions by offering reliability for patient engagement initiatives and better patient control in terms of healthcare data as well as enhanced data integration. The blockchain-based decentralized network is engaged in creating a marketplace for the vendors to manage and control the accessibility for health-related information in a trusted and secured manner. In addition to these, it also provides a secure platform to manage the consent of the patients to share their personal health-related information with pharma clinical trials.

Company:

Medable.

Medable focuses on using a decentralized clinical trial in order to get effective therapies for the patients in an efficient manner. The key products offered by the company include digital and DCT screening, TeleVisit, eConsent, decentralized trial platform, and remote patient monitoring, along with ePRO and eCOA. Medable has completed approximately 150 studies with its operating and distribution facilities in nearly 60 countries across the world (Medable, 2021).

Strategies adopted by Medable:

- In March 2018, Medable announced the launch of an "INSIGHT network", which is a blockchain-powered platform. The platform aligns incentives amid biopharmaceutical companies, medical researchers, and patients for self-directed real-world evidence (RWE) information sharing and allows for transparent and auditable exchange of medical information.

\section{Accessibility of Electronic Health Records}

\section{Problem:}

The electronic healthcare records (EHRs) refer to the electronic form of a patient's healthcare reports. The core 
components of these records comprise computerized physician order entry, administrative functions, pharmacy systems, radiology systems, clinical documentation, and lab systems (Seymour et al., 2014). Implementation of EHRs in the healthcare system enables easier organization and accessibility of the records. However, EHR of one patient's healthcare provider may vary from the same patient's healthcare provider, thereby posing to be difficult for managing the same.

Opportunities: Implementation of blockchain technology in the healthcare systems is expected to completely revolutionize the way patient's electronic health records are stored and shared. The technology offers safer mechanisms for the information exchange of medical data, by securing it over a distributed peer-to-peer system (Mayer et al., 2019).

Company:

CareCloud.

MTBC, a US-based company, is engaged in offering a suite of privately operated cloud-based solutions, to the hospitals and healthcare providers. The company's offerings include softwareas-a-service platform (SaaS), which comprises electronic health record (EHR), practice management (PM), telehealth, revenue cycle management (RCM), patient experience management (PXM), and business intelligence solutions for highperformance health groups.

The company is one of the leading providers of cloud-based healthcare IT services and solutions. The company's market presence and solutions are significantly gaining traction, with a team of nearly 40,000 healthcare providers. The company has its operating and distribution facilities across nearly 50 states, backed up by approximately 3,000 team members in different regions of the world.

Strategies adopted by CareCloud:

- In March 2021, MTBC announced that it has been renamed to "CareCloud".

- The company emphasizes on its expansion into new markets by offering powerful and innovative solutions to its clients.

\section{REFERENCES}

Ali, A., Rahim, H. A., Pasha, M. F., Dowsley, R., Masud, M., Ali, J., et al. (2021). Security, Privacy, and Reliability in Digital Healthcare Systems Using Blockchain. Electronics 10, 2034. doi:10.3390/electronics10162034

AlpVision SA (2021). Pharmaceutical Counterfeiting: What Are the Actual Risks. Switzerland: AlpVision SA. Available at: https://alpvision.com/career/.

Attaran, M. (2020). Blockchain Technology in Healthcare: Challenges and Opportunities. Int. J. Healthc. Manag. 14, 1-14. doi:10.1080/20479700.2020.1843887

Ben Fekih, R., and Lahami, M. (2020). "Application of Blockchain Technology in Healthcare: A Comprehensive Study," in Application of Blockchain Technology in Healthcare: A Comprehensive Study. The Impact of Digital Technologies on Public Health in Developed and Developing Countries (Cham: Springer), 268-276. doi:10.1007/978-3-030-51517-1_23

Blackstone, E. A., Fuhr, J. P., Jr, and Pociask, S. (2014). The Health and Economic Effects of Counterfeit Drugs. Am. Health Drug Benefits 7, 216-224.

Blockchains (2019). "Blockchain for Healthcare: Use Cases and Applications," in Blockchains 101. Available at: https://101blockchains.com/blockchain-forhealthcare/.

\section{CONCLUSION}

This study discusses about the different application areas of blockchain technology in the healthcare sector. Application of blockchain is expected to go above the range in the field of economics, with the fact that its potentiality largely depends upon its acceptability in the healthcare system. Blockchain is based on the concept of "append-only" open ledger, according to which every contract occurring within a setup, is added to the ledger, when verified by the connections in the system.

The article emphasizes on the fact that this technology is widely used to ensure the availability of patient-related information, enhance patient engagement, combine and coordinate the information from multiple providers, and enable secure communication between the providers and patients. Transparency in the information structure, distributed nature, and immutable record-keeping features of blockchain enable the businesses to easily minimize the costs and increase the efficiency.

However, uncertainty in cost, regulatory issues, technological and organizational issues, interoperability issues, and scaling are the few factors restraining the adoption of blockchain in healthcare businesses. Blockchain may not only be the remedy for all the healthcare challenges, but it certainly offers the potentiality to eliminate the mediator and optimize the workflow activities over the forecast period.

\section{DATA AVAILABILITY STATEMENT}

The original contributions presented in the study are included in the article/Supplementary Material; further inquiries can be directed to the corresponding author.

\section{AUTHOR CONTRIBUTIONS}

The author confirms being the sole contributor of this work and has approved it for publication.

CapGemini (2017). Blockchain: A Healthcare Industry View. United Kingdom: CapGemini.

Change Healthcare (2020). Leveraging Data and Insights. US: Change Healthcare.

CitiusTech (2018). Blockchain for Healthcare: An Opportunity to Address many Complex Challenges in Healthcare. Princeton, NJ: CitiusTech.

Cummings, S. (2019). The Four Blockchain Generations. Altcoin Mag.

FourWeekMBA (2021). "The Leading Source of Original Research and Insights on Business Model, S. D.," in Blockchain in Healthcare (FourWeekMBA). Available at: https://fourweekmba.com/blockchain-inhealthcare/.

Frost and Sullivan (2019). Global Blockchain Technology Market in the Healthcare Industry, 2018-2022. Santa Clara, CA: Frost \& Sullivan.

Haq, I., and Muselemu, O. (2018). Blockchain Technology in Pharmaceutical Industry to Prevent Counterfeit Drugs. Int. J. Comp. Appl. 180 (25), 8-12. doi:10.5120/ijca2018916579

Healthcare Information and Management Systems Society (2021). Blockchain in Healthcare. Chicago, IL: Healthcare Information and Management Systems Society, Inc.

Health Research Funding(2021). 20 Shocking Counterfeit Drugs Statistics. Available at: HealthResearchFunding.org. 
LeewayHertz (2021). Use Cases of Blockchain in Healthcare. San Francisco, CA: Blockchain in Healthcare.

Mayer, A. H., da Costa, C. A., and Righi, R. d. R. (2019). Electronic Health Records in a Blockchain: A Systematic Review. Health Inform. J 26 (2), 1273-1288. doi:10.1177/1460458219866350

Medable (2021). Research for Every Body and Every Biology. CA, United States: Medable.

MediBloc Limited (2021). A Decentralized Blockchain Network for Health Data Sovereignty and Interoperability. Gibraltar: MediBloc Limited.

Pharma IQ News (2011). Impact of Counterfeits on Pharmaceutical Industry Counterfeiting Poses Threats to Patient Safety. Available at: https://www. pharma-iq.com/market-access/press-releases/impact-of-counterfeits-onpharmaceutical-industry (Accessed March 10, 2011).

Onik, M. M. H., Aich, S., Yang, J., Kim, C.-S., and Kim, H.-C. (2019). "Blockchain in Healthcare: Challenges and Solutions," in Big Data Analytics for Intelligent Healthcare Management, 197-226. doi:10.1016/B978-0-12-818146-1. 00008-8

Prokofieva, M., and Miah, S. J. (2019). Blockchain in Healthcare. Australas. J. Inf. Syst. 23, 22. doi:10.3127/ajis.v23i0.2203

Seymour, T., Frantsvog, D., and Graeber, T. (2014). Electronic Health Records (EHR). Am. J. Health Sci. (Ajhs) 3 (3), 201-210.doi:10.19030/ ajhs.v3i3.7139
Singhal, S., Dash, P., Schneider, T., Chowdhary, S., and Aggarwal, H. (2019). For Better Healthcare Claims Management, Think "Digital First". Chicago: McKinsey \& Company.

Yadav, S. S. (2019). Blockchain - A Panacea for Healthcare Systems. HCL Technologies Limited - Digitalization.

Conflict of Interest: The author declares that the research was conducted in the absence of any commercial or financial relationships that could be construed as a potential conflict of interest.

Publisher's Note: All claims expressed in this article are solely those of the authors and do not necessarily represent those of their affiliated organizations, or those of the publisher, the editors, and the reviewers. Any product that may be evaluated in this article, or claim that may be made by its manufacturer, is not guaranteed or endorsed by the publisher.

Copyright (C) 2022 Thakur. This is an open-access article distributed under the terms of the Creative Commons Attribution License (CC BY). The use, distribution or reproduction in other forums is permitted, provided the original author(s) and the copyright owner(s) are credited and that the original publication in this journal is cited, in accordance with accepted academic practice. No use, distribution or reproduction is permitted which does not comply with these terms. 